PROCEEDINGS OF THE

AMERICAN MATHEMATICAL SOCIETY

Volume 139, Number 5, May 2011, Pages 1691-1706

S 0002-9939(2010)10613-5

Article electronically published on October 18, 2010

\title{
ON OPTIMAL ESTIMATES FOR THE LAPLACE-LERAY COMMUTATOR IN PLANAR DOMAINS WITH CORNERS
}

\author{
ELAINE COZZI AND ROBERT L. PEGO
}

(Communicated by Walter Craig)

\begin{abstract}
For smooth domains, Liu et al. (Comm. Pure Appl. Math. 60: 1443-1487, 2007) used optimal estimates for the commutator of the Laplacian and the Leray projection operator to establish well-posedness of an extended Navier-Stokes dynamics. In their work, the pressure is not determined by incompressibility, but rather by a certain formula involving the Laplace-Leray commutator. A key estimate of Liu et al. controls the commutator strictly by the Laplacian in $L^{2}$ norm at leading order. In this paper we show that this strict control fails in a large family of bounded planar domains with corners. However, when the domain is an infinite cone, we find that strict control may be recovered in certain power-law weighted norms.
\end{abstract}

\section{INTRODUCTION}

In this paper, we study estimates for $[\Delta, P]=\Delta P-P \Delta$, the commutator of the Laplacian and the Leray projection operator, in planar domains with corners. In a bounded domain $\Omega \subset \mathbb{R}^{N}$, the Leray projection operator $P$ is defined as follows: Given any $a \in L^{2}\left(\Omega, \mathbb{R}^{N}\right)$, there exists a unique $q \in H^{1}(\Omega)$ with $\int_{\Omega} q=0$ and such that $P a:=a+\nabla q$ satisfies

$$
0=\langle P a, \nabla \phi\rangle=\langle a+\nabla q, \nabla \phi\rangle
$$

for all $\phi \in H^{1}(\Omega)$. In [3], Liu et al. proved the following $L^{2}$-estimate for the commutator of the Leray projection operator and the Laplacian.

Theorem 1.1. Let $\Omega$ be a connected, bounded domain in $\mathbb{R}^{N}, N \geq 2$, with $C^{3}$ boundary. For any $\beta>\frac{1}{2}$, there exists $C \geq 0$ such that for all vector fields $u \in$ $H^{2} \cap H_{0}^{1}\left(\Omega, \mathbb{R}^{N}\right)$,

$$
\int_{\Omega}|[\Delta, P] u|^{2} \leq \beta \int_{\Omega}|\Delta u|^{2}+C \int_{\Omega}|\nabla u|^{2} .
$$

Theorem 1.1 has significant applications to the Navier-Stokes equations. We recall that on a bounded domain $\Omega$ in $\mathbb{R}^{N}$ for $N \geq 2$, the Navier-Stokes equations

Received by the editors December 18, 2009 and, in revised form, May 19, 2010.

2010 Mathematics Subject Classification. Primary 35-XX; Secondary 76-XX.

This material is based upon work supported by the National Science Foundation under Grants No. DMS06-04420 and DMS09-05723 and partially supported by the Center for Nonlinear Analysis (CNA) under National Science Foundation Grant No. DMS06-35983.

(C)2010 American Mathematical Society Reverts to public domain 28 years from publication 
modeling incompressible viscous fluid flow with no-slip boundary conditions are given by

$$
\left\{\begin{array}{c}
\partial_{t} u+u \cdot \nabla u+\nabla p=\nu \Delta u+f, \\
\nabla \cdot u=0 \\
\left.u\right|_{\Gamma}=0
\end{array}\right.
$$

where $\Gamma=\partial \Omega, u$ denotes the velocity of the fluid, $p$ denotes the pressure, and $\nu$ represents the viscosity. In [3], the authors consider strong solutions to $(N S)$ with constant $\nu>0$ and show that the pressure satisfies

$$
\nabla p=(I-P)(f-u \cdot \nabla u)+\nu[\Delta, P] u .
$$

For such solutions they prove the unconditional stability and convergence of a simple time discretization scheme which decouples the updates of velocity and pressure. The decoupling of these variables is significant in that it eliminates the need for an inf-sup condition which is often necessary to prove the stability in finite-element schemes. A critical ingredient in the proof of stability in [3] is that by invoking Theorem 1.1 with $\beta<1$, one can strictly control the pressure gradient by the viscosity term plus lower-order terms. As a result, Liu et al. establish the wellposedness of an extended Navier-Stokes dynamics in which the pressure $p$ is always determined by the formula (1.3) and the zero-divergence condition is dropped in general. We refer the reader to [3] for further details and discussion.

Theorem 1.1 assumes that the boundary $\Gamma$ of $\Omega$ is $C^{3}$. One would like to weaken this assumption to allow, for example, sharp corners on $\Gamma$. In this paper, we show that such an improvement is not possible. We let $\mathcal{K}_{\sigma}$ denote an infinite cone centered at the origin, taking the form

$$
\mathcal{K}_{\sigma}=\left\{\left(x_{1}, x_{2}\right) \in \mathbb{R}^{2}: 0<r<\infty, 0<\theta<\sigma\right\},
$$

where $r$ and $\theta$ denote the polar coordinates of $\left(x_{1}, x_{2}\right)$ and $\sigma \in(0,2 \pi)$. We consider bounded domains $\Omega \subset \mathbb{R}^{2}$ satisfying the following property: there is a neighborhood $U$ of 0 such that $U \cap \tilde{\Omega}=U \cap \mathcal{K}_{\sigma}$ for some rotated translate $\tilde{\Omega}=R\left(\Omega-x_{0}\right)$ of $\Omega$ and for some $\sigma \neq \pi$. In this case we call $\Omega$ a bounded domain with a straight corner. We claim that Theorem 1.1 fails on any such domain.

Theorem 1.2. Let $\Omega$ in $\mathbb{R}^{2}$ be a bounded domain with a straight corner. Then for every $\beta<1$ and for every $C \in \mathbb{R}$, there is a vector field $u \in H^{2} \cap H_{0}^{1}\left(\Omega, \mathbb{R}^{2}\right)$ satisfying

$$
\int_{\Omega}|[\Delta, P] u|^{2}>\beta \int_{\Omega}|\Delta u|^{2}+C \int_{\Omega}|\nabla u|^{2} .
$$

While $\beta<1$ is not possible in general for a bounded domain with a straight corner, a recent paper of Rostamian and Soane [4] suggests that, for such domains, an estimate similar to (1.2) with $\beta<1$ may hold in weighted Sobolev spaces. In [4, the authors reformulate the time discretization scheme of [3] in nonconvex polygonal domains using such weighted spaces. While the authors do not prove convergence of their scheme, they do give numerical evidence suggesting that this scheme converges to the correct solution.

We are motivated by [4] and elliptic regularity theory with weights [2] to allow for corners on $\Gamma$ and look to prove an optimal estimate similar to (1.2) in a weighted $L^{2}$-space. For the most part, we study conical domains of the form shown in (1.4). The weighted spaces considered in [2] are defined as follows. 
Definition 1.3. For an integer $l \geq 0$ and a real number $\alpha$, we define the space $V_{2, \alpha}^{l}\left(\mathcal{K}_{\sigma}\right)$ to be the closure of $C_{c}^{\infty}\left(\overline{\mathcal{K}}_{\sigma} \backslash\{0\}\right)$ with respect to the (scale-invariant) norm

$$
\|f\|_{V_{2, \alpha}^{l}}=\left(\int_{\mathcal{K}_{\sigma}} \sum_{|\rho| \leq l} r^{2(\alpha-l+|\rho|)}\left|D_{x}^{\rho} f\right|^{2} r d r d \theta\right)^{\frac{1}{2}}<\infty .
$$

We refer the reader to 2 for a more thorough discussion of weighted Sobolev spaces in an infinite cone.

Before we state the main theorem, we must define the Leray projection operator on unbounded domains. This definition differs from that given in (1.1), because if $\Omega$ is unbounded, then $\nabla H^{1}(\Omega)$ is not closed in $L^{2}(\Omega)$. To remedy this, we fix a bounded domain $B \subset \Omega \subset \mathbb{R}^{N}$, and we define the space

$$
Y=\left\{q \in L_{l o c}^{2}(\Omega): \nabla q \in L^{2}\left(\Omega, \mathbb{R}^{N}\right) \text { and } \int_{B} q=0\right\} .
$$

Then $Y$ is a Hilbert space with norm $\|q\|_{Y}^{2}=\int_{\Omega}|\nabla q|^{2}$, and the space $\nabla Y$ is closed in $L^{2}\left(\Omega, \mathbb{R}^{N}\right)$. We define the Leray projection operator $P$ as in (1.1), except that we assume $q$ is in $Y(\Omega)$ instead of $H^{1}(\Omega)$. Further discussion of the Leray projection operator on unbounded domains can be found in [5].

We remark that if $\Omega$ is Lipschitz, $C_{c}^{\infty}(\bar{\Omega})$ is dense in $Y$. The proof of this fact is similar to the proof for $\Omega=\mathbb{R}_{+}^{N}$ indicated in [3], based on the case $\Omega=\mathbb{R}^{N}$ treated in [5, Lemma 2.5.4].

We are now prepared to state the main theorem.

Theorem 1.4. Suppose $\sigma \in(0,2 \pi)$ and let $\mathcal{K}_{\sigma}$ be an infinite planar cone as in (1.4). Let $\alpha \neq 1$. Then the following estimate holds for all $u \in C_{c}^{\infty}\left(\overline{\mathcal{K}}_{\sigma} \backslash\{0\}, \mathbb{R}^{2}\right)$ :

$$
\int_{\mathcal{K}_{\sigma}} r^{2 \alpha}|[\Delta, P] u|^{2} r d r d \theta \leq \beta_{\sigma, \alpha} \int_{\mathcal{K}_{\sigma}} r^{2 \alpha}|\Delta u|^{2} r d r d \theta
$$

where

$$
\beta_{\sigma, \alpha}=\sup _{k>0} \max \left\{\hat{\beta}_{+, k}, \hat{\beta}_{-, k}\right\}
$$

with

$$
\hat{\beta}_{ \pm, k}=\frac{k^{2}+\alpha^{2}}{2 k^{2}(1-\alpha)}\left(1-e^{-2 k \sigma}\right) \Re\left\{\frac{(1-\alpha+i k)\left(1 \pm e^{-(k+i \alpha-2 i) \sigma}\right)}{\left(1 \pm e^{-(k-i \alpha) \sigma}\right)\left(1-e^{-2(k+i \alpha-i) \sigma}\right)}\right\} .
$$

Moreover, $\beta_{\sigma, \alpha}$ is the smallest constant satisfying (1.8) for every

$$
u \in C_{c}^{\infty}\left(\overline{\mathcal{K}}_{\sigma} \backslash\{0\}, \mathbb{R}^{2}\right) .
$$

We will prove Theorem 1.4 in Sections 2 and 3 , In Section 5, we show that Theorem 1.4 implies Theorem 1.2 .

The expressions in (1.9) are sufficiently complicated so that it is difficult to characterize exactly when $\beta_{\sigma, \alpha}<1$ holds. We will make a few observations, however, and provide numerical evidence which suggests that for all $\sigma \in(0,2 \pi)$ except for one value $\sigma=\sigma_{c} \approx 1.4303 \pi$, we have $\beta_{\sigma, \alpha}<1$ for $\alpha$ in some interval just to the left or right of $\alpha=0$.

First, note that as $k \rightarrow \infty$ we have $\hat{\beta}_{ \pm, k} \rightarrow \frac{1}{2}$. For $\alpha=0$ we compute that

$$
\hat{\beta}_{ \pm, k}=\frac{1}{2} \frac{\cosh ^{2} k \sigma-\cos ^{2} \sigma \mp \cosh k \sigma \sin ^{2} \sigma \mp k \sin \sigma \cos \sigma \sinh k \sigma}{\cosh ^{2} k \sigma-\cos ^{2} \sigma},
$$


from which we see that if $\sigma=\pi$, then $\hat{\beta}_{ \pm, k} \equiv \frac{1}{2}$; hence $\beta_{\pi, 0}=\frac{1}{2}$. This half-space estimate (1.8) with constant weight was already proved in [3] and explains why the condition $\beta>\frac{1}{2}$ is essentially optimal in Theorem 1.1. Note that due to the dilation invariance of the domain, no lower-order term such as that in (1.2) should appear in the half-space case, since it would scale differently under dilation.

Whenever $\pi \neq \sigma \in(0,2 \pi)$, however, we have $\hat{\beta}_{-, 0}=1, \hat{\beta}_{+, 0}=0$. Thus, whenever the weight is constant $(\alpha=0)$ and the cone has a corner $(\sigma \neq \pi)$, we conclude that the optimal constant $\beta_{\sigma, 0} \geq 1$. Our proof of Theorem 1.2 relies on this fact.

It is easy to approximate $\beta_{\sigma, \alpha}$ numerically. For a number of values of the cone angle $\sigma$, in Figure 1 we plot $\log _{10} \beta_{\sigma, \alpha}$ vs. $\alpha$ for $\alpha \in[-1,1]$. Spikes appear in many of these graphs, providing evidence of singularities where presumably $\beta_{\sigma, \alpha}=+\infty$. After closer examination, these graphs suggest that:

- $\beta_{\sigma, \alpha}=1$ whenever $\alpha=0$ and $\sigma \neq \pi$.

- $\beta_{\sigma, \alpha}<1$ for small $\alpha>0$ when $0<\sigma<\pi$ or $\sigma_{c}<\sigma<2 \pi$.

- $\beta_{\sigma, \alpha}<1$ for small $\alpha<0$ when $\pi<\sigma<\sigma_{c}$.

The number $\sigma_{c} \approx 1.4303 \pi$ satisfying $\sigma_{c} \cot \sigma_{c}-1=0$ appears to be a critical value of $\sigma$ where the minimum of $\beta_{\sigma, \alpha}$ occurs at $\alpha=0$ and the minimum value is 1 . To see this, we observe that numerical evidence indicates that for $\sigma$ near the critical value and for $\alpha$ near $0, \beta_{\sigma, \alpha}$ is achieved at $k=0$. We therefore take the limit as $k$ approaches 0 of $\hat{\beta}_{+, k}$ and $\hat{\beta}_{-, k}$, which yields the formulas for $\hat{\beta}_{+, 0}$ and $\hat{\beta}_{-, 0}$ given in (4.2) and (4.3). Numerical evidence again shows that for $\sigma$ in a neighborhood of the critical value and for $\alpha$ near $0, \hat{\beta}_{+, 0}>\hat{\beta}_{-, 0}$. Using Maple to differentiate $\hat{\beta}_{+, 0}$ with respect to $\alpha$ and evaluating the derivative at $\alpha=0$, we find that

$$
\left.\partial_{\alpha} \hat{\beta}_{+, 0}\right|_{\alpha=0}=\sigma \cot \sigma-1 .
$$

These numerical results also suggest that $\beta_{\sigma, \alpha}<1$ for convex cones, uniformly in $\sigma$ for positive $\alpha$ in a fixed interval. So for a bounded polygonal domain $\Omega$ that is convex, say, we conjecture that an inequality like

$$
\int_{\Omega} r^{2 \alpha}|[\Delta, P] u|^{2} \leq \int_{\Omega} r^{2 \alpha}\left(\beta|\Delta u|^{2}+C|\nabla u|^{2}\right)
$$

will hold for some $\beta<1$ and $C$ independent of $u$ in a suitable space of functions vanishing on $\partial \Omega$, provided $\alpha$ is small and positive. Here $r=r(x)$ would be the distance from $x \in \Omega$ to the nearest corner on $\Gamma$. The last term on the right hand side of (1.12) comes from the definition of the $V_{2, \alpha}^{2}$ norm on a bounded domain [2], given by

$$
\|u\|_{V_{2, \alpha}^{2}(\Omega)}=\left(\int_{\Omega} r^{2 \alpha} \sum_{|\rho| \leq 2}\left|D_{x}^{\rho} u\right|^{2} d x\right)^{\frac{1}{2}} .
$$

We do not include the term $\left\|r^{\alpha} u\right\|_{L^{2}}^{2}$ on the right hand side of (1.12), because it can be controlled by first order partial derivatives using a Hardy inequality [2].

A proof of (1.12) could have interesting implications. In particular, one may be able to use (1.12) to prove unconditional stability and convergence of the time discretization scheme of Liu et al. in convex polygonal domains for $u$ in the appropriate weighted space. This proof could, in turn, lead to well-posedness results for the unconstrained formulation of $(N S)$ in polygonal domains. However, we have no proof of (1.12) at this time. 

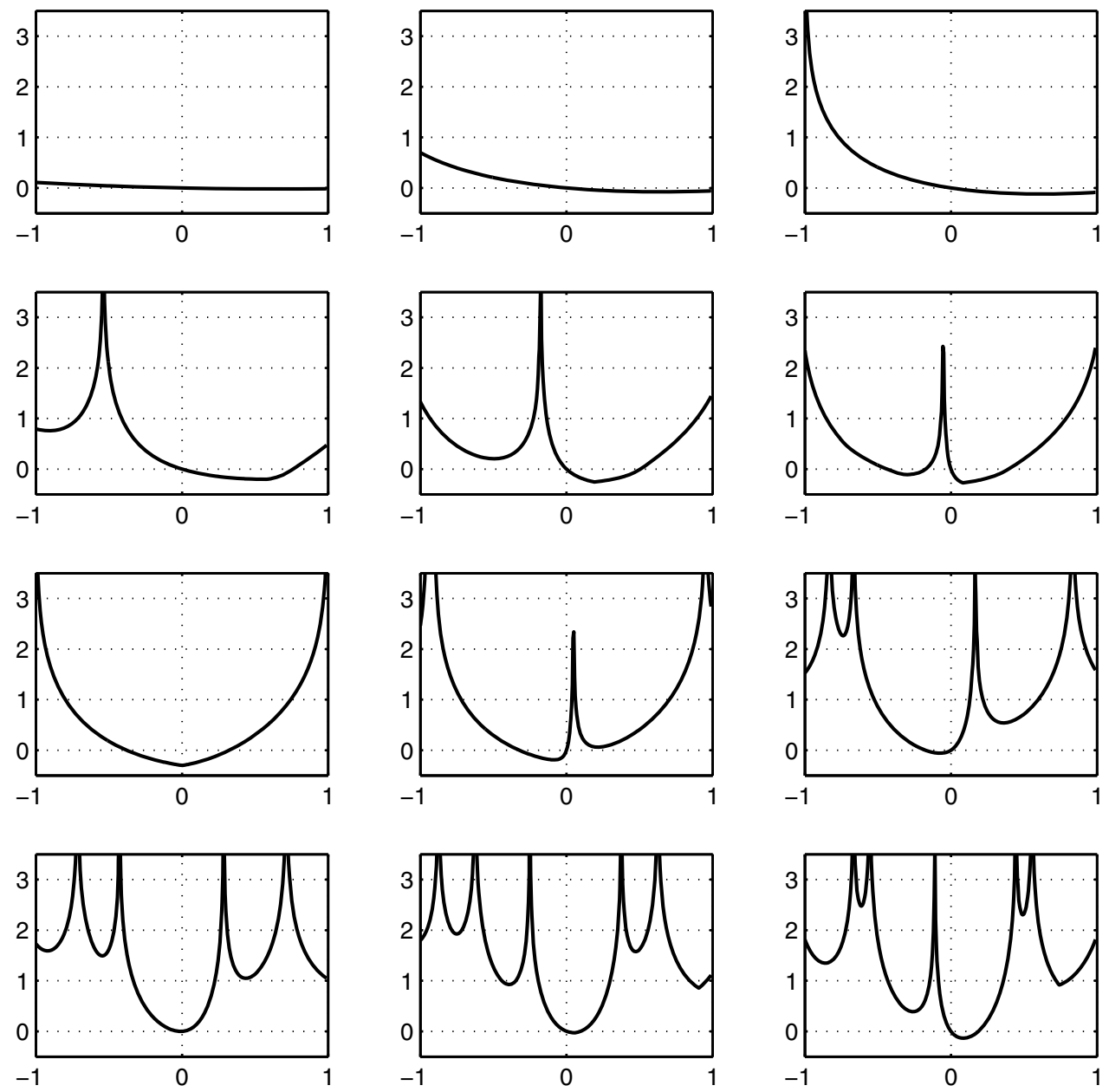

FiguRE $1 . \log _{10}\left(\beta_{\sigma, \alpha}\right)$ vs. $\alpha$ for various $\sigma$. From left to right, top to bottom, $\sigma / \pi=0.2,0.4,0.5,0.65,0.85,0.95,1,1.05,1.2,1.4,1.6,1.8$.

\section{Preliminary transform in RAdiUS}

From the pressure formula (1.3) we see that the commutator $[\Delta, P] u$ represents the contribution of the viscosity term to the Navier-Stokes pressure gradient. Specifically, $[\Delta, P] u$ represents the pressure gradient for the linear Stokes equations with no-slip boundary and without forcing. For this reason, as in [3], we refer to the corresponding pressure as the Stokes pressure, denoted $p_{\mathrm{S}}=p_{\mathrm{S}}(u)$. From (1.1), when $a=u \in H^{2}(\Omega)$ with $\Omega$ unbounded, we have $\nabla \Delta q=\Delta \nabla q=\nabla \nabla \cdot a$, and it follows easily (as in [3]) that

$$
[\Delta, P] u=(I-P)(\Delta u-\nabla \nabla \cdot u)=\nabla p_{\mathrm{S}} .
$$

We recall from [3, Sec. 2.1] that the Stokes pressure $p_{\mathrm{S}}$ is determined (up to constant) as the solution to the boundary value problem

$$
\Delta p_{\mathrm{S}}=0 \quad \text { in } \Omega, \quad n \cdot \nabla p_{\mathrm{S}}=n \cdot(\Delta-\nabla \nabla \cdot) u \quad \text { on } \Gamma .
$$


(The boundary condition holds in $H^{-1 / 2}(\Gamma)$ due to a standard trace theorem, since the vector fields $\Delta u-\nabla \nabla \cdot u$ and $\nabla p_{\mathrm{S}}$ are in $L^{2}\left(\Omega, \mathbb{R}^{N}\right)$ with zero divergence.)

Letting

$$
\begin{gathered}
I_{p}=\left\|\nabla p_{\mathrm{S}}\right\|_{V_{2, \alpha}^{0}}^{2}=\int_{\mathcal{K}_{\sigma}} r^{2 \alpha}\left|\nabla p_{\mathrm{S}}\right|^{2} r d r d \theta, \\
I_{u}=\|\Delta u\|_{V_{2, \alpha}^{0}}^{2}=\int_{\mathcal{K}_{\sigma}} r^{2 \alpha}|\Delta u|^{2} r d r d \theta,
\end{gathered}
$$

we see that in order to prove Theorem 1.4, we must determine the smallest constant $\beta_{\sigma, \alpha}$ satisfying the inequality $I_{p} \leq \beta_{\sigma, \alpha} I_{u}$, subject to (2.1). In this section, we perform the first steps in our attempt to find $\beta_{\sigma, \alpha}$. These steps amount to taking a Mellin transform of the problem. We first rewrite $I_{p}, I_{u}$ and (2.1) in terms of the polar coordinates $(r, \theta)$, then change variables using $r=e^{s}$, which transforms $\mathcal{K}_{\sigma}$ to an infinite strip $\mathcal{S}$. Taking a Fourier transform will reduce the problem to a family of maximization problems parametrized by a Fourier variable $k \in \mathbb{R}$.

2.1. We begin by letting

$$
J=\left(\begin{array}{cc}
0 & -1 \\
1 & 0
\end{array}\right), \quad e^{J \theta}=\left(\begin{array}{cc}
\cos \theta & -\sin \theta \\
\sin \theta & \cos \theta
\end{array}\right) .
$$

A straightforward calculation shows that

$$
\nabla p_{\mathrm{S}}=r^{-1} e^{J \theta}\left(\begin{array}{c}
r \partial_{r} p_{\mathrm{S}} \\
\partial_{\theta} p_{\mathrm{S}}
\end{array}\right)
$$

allowing us to rewrite $I_{p}$ as

$$
I_{p}=\int_{\mathcal{K}_{\sigma}}\left(\left|r \partial_{r} p_{\mathrm{S}}\right|^{2}+\left|\partial_{\theta} p_{\mathrm{S}}\right|^{2}\right) r^{2 \alpha-1} d r d \theta .
$$

We change variables by letting $r=e^{s}$, resulting in a transformation of the domain $\mathcal{K}_{\sigma}$ to an infinite strip $\mathcal{S}=\left\{(s, \theta) \in \mathbb{R}^{2}:-\infty<s<\infty, 0<\theta<\sigma\right\}$. We then let $q=e^{\alpha s} p_{\mathrm{S}}$ and express $I_{p}$ in terms of $q$. We conclude that

$$
I_{p}=\int_{\mathcal{S}}\left(\left|\partial_{s} q-\alpha q\right|^{2}+\left|\partial_{\theta} q\right|^{2}\right) d s d \theta=\int_{-\infty}^{\infty} I_{p, k} d k
$$

where $k$ is the Fourier variable corresponding to $s$, and

$$
I_{p, k}=\int_{0}^{\sigma}\left(|(k+i \alpha) \hat{q}|^{2}+\left|\partial_{\theta} \hat{q}\right|^{2}\right) d \theta .
$$

2.2. To rewrite $I_{u}$, we first calculate

$$
\Delta u=\nabla \cdot \nabla u=\left(r \partial_{r}+2\right)\left(r^{-1} \partial_{r} u\right)+\partial_{\theta}\left(r^{-2} \partial_{\theta} u\right)=r^{-2}\left(\left(r \partial_{r}\right)^{2}+\partial_{\theta}^{2}\right) u .
$$

If we let $u=r e^{J \theta} v$, we can show that

$$
\Delta u=r^{-1} e^{J \theta}\left(\left(r \partial_{r}+1\right)^{2} v+\left(\partial_{\theta}+J\right)^{2} v\right) .
$$

We again change variables to express $I_{u}$ as an integral over $\mathcal{S}$. We let $w=e^{s \alpha} v$, and we find that

$$
\begin{aligned}
I_{u} & =\int_{\mathcal{S}} e^{2 s \alpha}\left|\left(\partial_{s}+1\right)^{2} v+\left(\partial_{\theta}+J\right)^{2} v\right|^{2} d s d \theta \\
& =\int_{\mathcal{S}}\left|\left(\partial_{s}+1-\alpha\right)^{2} w+\left(\partial_{\theta}+J\right)^{2} w\right|^{2} d s d \theta=\int_{-\infty}^{\infty} I_{u, k} d k,
\end{aligned}
$$


where

$$
I_{u, k}=\int_{0}^{\sigma}\left|(i k+1-\alpha)^{2} \hat{w}+\left(\partial_{\theta}+J\right)^{2} \hat{w}\right|^{2} d \theta
$$

2.3. As with $I_{p}$ and $I_{u}$, we wish to rewrite (2.1) in terms of $k, \theta, q$, and $w$. We perform a change of variables and rewrite the first condition of (2.1) as

$$
e^{-2 s}\left(\partial_{s}^{2}+\partial_{\theta}^{2}\right) p=0 \text {. }
$$

Recalling that $q=e^{s \alpha} p$, we find that $\Delta q-e^{-2 s}\left(2 \alpha \partial_{s}-\alpha^{2}\right) q=0$, so $\left(\partial_{\theta}^{2}+\partial_{s}^{2}-\right.$ $\left.2 \alpha \partial_{s}+\alpha^{2}\right) q=0$. To rewrite the boundary condition of (2.1), we observe that the left hand side can be rewritten using the equalities $\left\langle e_{\theta}, \nabla p_{\mathrm{S}}\right\rangle=\left\langle e_{2}, r^{-1}\left(r \partial_{r} p_{\mathrm{S}}, \partial_{\theta} p_{\mathrm{S}}\right)\right\rangle=$ $r^{-1} \partial_{\theta} p_{\mathrm{S}}$. For the right hand side, we use (2.4), combined with the equality

$$
\nabla \nabla \cdot u=r^{-1} e^{J \theta}\left(\begin{array}{c}
r \partial_{r} \nabla \cdot u \\
\partial_{\theta} \nabla \cdot u
\end{array}\right)=r^{-1} e^{J \theta}\left(\begin{array}{c}
r \partial_{r}\left(\left(r \partial_{r}+2\right) v_{1}+\partial_{\theta} v_{2}\right) \\
\partial_{\theta}\left(\left(r \partial_{r}+2\right) v_{1}+\partial_{\theta} v_{2}\right)
\end{array}\right)
$$

and the property $v=0$ when $\theta=0$ and $\theta=\sigma$, to conclude that

$\left\langle e_{\theta}, \Delta u-\nabla \nabla \cdot u\right\rangle=r^{-1}\left(\left(r \partial_{r}\right)^{2}+2 r \partial_{r}\right) v_{2}-\partial_{\theta} \partial_{r} v_{1}=-r^{-2} v_{2}-\partial_{\theta} \partial_{r} v_{1}=-\partial_{\theta} \partial_{r} v_{1}$ for $\theta=0$ and $\theta=\sigma$. We can therefore rewrite the boundary condition in (2.1) as

$$
\partial_{\theta} p_{\mathrm{S}}=-\partial_{s} \partial_{\theta} v_{1} .
$$

Using the equality $w=e^{s \alpha} v$, we see after a calculation that we can recast (2.1) as the following boundary value problem on $\mathcal{S}$ :

$$
\begin{aligned}
& \left(\partial_{\theta}^{2}+\partial_{s}^{2}-2 \alpha \partial_{s}+\alpha^{2}\right) q=0 \quad \text { in } \mathcal{S}, \\
& \partial_{\theta} q=-\partial_{\theta}\left(\partial_{s}-\alpha\right) w_{1} \quad \text { when } \theta=0, \sigma .
\end{aligned}
$$

Finally, taking the Fourier transform of (2.8) in $s$, we have that for each $k \in \mathbb{R}, \hat{q}$ must solve the boundary value problem

$$
\begin{aligned}
& \partial_{\theta}^{2} \hat{q}=(k+i \alpha)^{2} \hat{q} \quad \text { for } 0<\theta<\sigma \\
& \partial_{\theta} \hat{q}=-\partial_{\theta}(i k-\alpha) \hat{w}_{1} \quad \text { when } \theta=0, \sigma .
\end{aligned}
$$

\section{Optimization in ANGLE}

In this section, we determine $\beta_{\sigma, \alpha}=\sup \frac{I_{p}}{I_{u}}$ subject to (2.9) and the no-slip boundary condition. First, for $k \neq 0$ we suppress the $\alpha$ and $\sigma$ variables and define

$$
\beta_{k}=\sup \left\{\frac{I_{p, k}}{I_{u, k}}:(2.9) \text { holds, and } \hat{w}=0 \text { for } \theta=0, \sigma\right\} \text {. }
$$

Note that since $w$ is real, we have $\hat{w}(-k, \theta)=\overline{\hat{w}(k, \theta)}$; hence $I_{u,-k}=I_{u, k}$ from (2.6), and similarly $I_{p,-k}=I_{p, k}$ from (2.3). We conclude that $\beta_{k}$ is even in $k$. We define $\hat{\beta}_{\sigma, \alpha}=\sup _{k>0} \beta_{k}$, and we observe that

$$
I_{p}=\int_{-\infty}^{\infty} I_{p, k} d k \leq \int_{-\infty}^{\infty} \beta_{k} I_{u, k} d k \leq \hat{\beta}_{\sigma, \alpha} I_{u}
$$

We will prove Theorem 1.4 by computing that $\beta_{k}=\max \left\{\hat{\beta}_{+, k}, \hat{\beta}_{-, k}\right\}$, as given by (1.9), and by showing that $\hat{\beta}_{\sigma, \alpha} \leq \beta_{\sigma, \alpha}$. Since evidently $\hat{\beta}_{\sigma, \alpha} \geq \beta_{\sigma, \alpha}$, the result will follow. 
3.1. We first rewrite the quantity $I_{u, k}$ from (2.6) to diagonalize the matrix involved. We define

$$
V=\left(\begin{array}{cc}
1 & 1 \\
i & -i
\end{array}\right), \quad \Lambda=\left(\begin{array}{cc}
-1 & 0 \\
0 & 1
\end{array}\right) \text {. }
$$

Then letting $-i \hat{w}=V y$ with $y=\left(y_{1}, y_{2}\right)$, and using $J V=V(i \Lambda)$, we rewrite $I_{u, k}$ in the following way:

$$
\begin{aligned}
I_{u, k} & =\int_{0}^{\sigma}\left|\left((i k+1-\alpha)^{2}+\left(\partial_{\theta}^{2}+2 J \partial_{\theta}-1\right)\right)(V y)\right|^{2} d \theta \\
& =2 \int_{0}^{\sigma}\left|\left((i k+1-\alpha)^{2}+\left(\partial_{\theta}^{2}+2 i \Lambda \partial_{\theta}-1\right)\right) y\right|^{2} d \theta \\
& =2 \int_{0}^{\sigma}\left(\left|L_{1} y_{1}\right|^{2}+\left|L_{2} y_{2}\right|^{2}\right) d \theta
\end{aligned}
$$

where

$$
\begin{aligned}
& L_{1}=(i k+1-\alpha)^{2}+\partial_{\theta}^{2}-2 i \partial_{\theta}-1 \\
& L_{2}=(i k+1-\alpha)^{2}+\partial_{\theta}^{2}+2 i \partial_{\theta}-1 .
\end{aligned}
$$

3.2. We next express the quantity $I_{p, k}$ from (2.3) in terms of the boundary data from (2.9). From (2.9) it is clear that explicitly

$$
\begin{aligned}
& \hat{q}(k, \theta)=\alpha_{+} e^{(k+i \alpha)(\theta-\sigma)}+\alpha_{-} e^{-(k+i \alpha) \theta} \\
& \partial_{\theta} \hat{q}(k, \theta)=(k+i \alpha) \alpha_{+} e^{(k+i \alpha)(\theta-\sigma)}-(k+i \alpha) \alpha_{-} e^{-(k+i \alpha) \theta}
\end{aligned}
$$

for some complex constants $\alpha_{+}$and $\alpha_{-}$. If we define

$$
\omega=e^{-(k+i \alpha) \sigma}
$$

for convenience, we see from (3.4) that

$$
\hat{q}(k, \theta)= \begin{cases}\alpha_{+}+\alpha_{-} \omega, & \theta=\sigma \\ \alpha_{+} \omega+\alpha_{-}, & \theta=0\end{cases}
$$

and

$$
\partial_{\theta} \hat{q}(k, \theta)= \begin{cases}(k+i \alpha)\left(\alpha_{+}-\alpha_{-} \omega\right), & \theta=\sigma, \\ (k+i \alpha)\left(\alpha_{+} \omega-\alpha_{-}\right), & \theta=0 .\end{cases}
$$

Combining (3.7) with the equality $-i \hat{w}_{1}=(V y)_{1}$, we can rewrite the boundary conditions in (2.9) as

$$
\begin{array}{ll}
\alpha_{+}-\alpha_{-} \omega=\partial_{\theta}\left(y_{1}+y_{2}\right), & \theta=\sigma, \\
\alpha_{+} \omega-\alpha_{-}=\partial_{\theta}\left(y_{1}+y_{2}\right), & \theta=0 .
\end{array}
$$

These equations will be used later to determine $\alpha_{+}$and $\alpha_{-}$from $y$ (note $\omega^{2} \neq 1$ ). To rewrite $I_{p, k}$, we apply (2.9) and integrate by parts. This gives

$$
\begin{aligned}
& \int_{\mathcal{S}}\left|\left(\partial_{s}-\alpha\right) q\right|^{2} d s d \theta=\int_{\mathcal{S}}(k+i \alpha) \hat{q}(k-i \alpha) \overline{\hat{q}} d k d \theta \\
& =\int_{-\infty}^{\infty}\left(\partial_{\theta} \hat{q}(\sigma) \overline{\hat{q}}(\sigma)-\partial_{\theta} \hat{q}(0) \overline{\hat{q}}(0)\right) d k-\int_{\mathcal{S}} \partial_{\theta} \hat{q} \partial_{\theta} \overline{\hat{q}} d k d \theta-\int_{\mathcal{S}} 2\left(i k \alpha-\alpha^{2}\right) \hat{q} \hat{q} d k d \theta,
\end{aligned}
$$

which, in light of (2.2), allows us to write

$$
I_{p, k}=\partial_{\theta} \hat{q}(\sigma) \overline{\hat{q}}(\sigma)-\partial_{\theta} \hat{q}(0) \overline{\hat{q}}(0)-\int_{0}^{\sigma} 2\left(i k \alpha-\alpha^{2}\right) \hat{q} \overline{\hat{q}} d \theta .
$$


In order to write $\int_{0}^{\sigma} 2\left(i k \alpha-\alpha^{2}\right) \hat{q} \bar{q} d \theta$ in terms of $\alpha_{+}$and $\alpha_{-}$, we use (3.4) to evaluate the dot product and integrate. We conclude that

$$
\begin{aligned}
& \int_{0}^{\sigma} 2\left(i k \alpha-\alpha^{2}\right) \hat{q} \hat{\hat{q}} d \theta=\left(i k \alpha-\alpha^{2}\right)\left(\frac{\left|\alpha_{+}\right|^{2}}{k}+\frac{\left|\alpha_{-}\right|^{2}}{k}\right)\left(1-e^{-2 k \sigma}\right) \\
& \quad+(i k-\alpha)\left(i \alpha_{-} \bar{\alpha}_{+}+i \alpha_{+} \bar{\alpha}_{-}\right) e^{-(k+i \alpha) \sigma}\left(1-e^{2 i \alpha \sigma}\right) \\
& =\left(i k \alpha-\alpha^{2}\right)\left(\frac{\left|\alpha_{+}\right|^{2}}{k}+\frac{\left|\alpha_{-}\right|^{2}}{k}\right)\left(1-|\omega|^{2}\right)+(k+i \alpha)\left(\alpha_{-} \bar{\alpha}_{+}+\alpha_{+} \bar{\alpha}_{-}\right)(\bar{\omega}-\omega) .
\end{aligned}
$$

Similarly, to compute $\partial_{\theta} \hat{q}(\sigma) \overline{\hat{q}}(\sigma)-\partial_{\theta} \hat{q}(0) \overline{\hat{q}}(0)$, we use the formulas for $\hat{q}$ and $\partial_{\theta} \hat{q}$ on the boundary given in (3.6) and (3.7) to write

$$
\begin{gathered}
\partial_{\theta} \hat{q}(\sigma) \overline{\hat{q}}(\sigma)-\partial_{\theta} \hat{q}(0) \overline{\hat{q}}(0)=(k+i \alpha)\left\{\left(\left|\alpha_{+}\right|^{2}+\left|\alpha_{-}\right|^{2}\right)\left(1-|\omega|^{2}\right)\right. \\
\left.+\left(\alpha_{+} \bar{\alpha}_{-}+\alpha_{-} \bar{\alpha}_{+}\right)(\bar{\omega}-\omega)\right\} .
\end{gathered}
$$

Plugging (3.10) and (3.11) into (3.9), we discover that

$$
I_{p, k}=\left(\frac{k^{2}+\alpha^{2}}{k}\right)\left(\left|\alpha_{+}\right|^{2}+\left|\alpha_{-}\right|^{2}\right)\left(1-|\omega|^{2}\right) .
$$

3.3. By the results of the previous subsection, $\beta_{k}$ is the supremum of the ratio $I_{p, k} / I_{u, k}$ subject to (3.8) and the no-slip boundary conditions $y=0$ for $\theta=0, \sigma$. In order to compute $\beta_{k}$, we will argue that the supremum in (3.1) is a maximum and use a variational argument.

The existence of a maximizer is proved by a standard argument in the calculus of variations: It is clear that $0<\beta_{k} \leq \infty$ and that the ratio $I_{p, k} / I_{u, k}$ is a homogeneous function of $y$. Thus we may choose a maximizing sequence of vector functions $y$ with fixed $H^{2}$ Sobolev norm on $[0, \sigma]$. Evidently, the quantities $L_{1} y_{1}, L_{2} y_{2}$ remain bounded in $L^{2}$, and the complex scalar quantities $\partial_{\theta} y_{1}, \partial_{\theta} y_{2}$ at $\theta=0, \sigma$ remain bounded. We may choose a subsequence converging weakly in $H^{2}$ such that the quantities $\partial_{\theta} y_{1}, \partial_{\theta} y_{2}$ at $\theta=0, \sigma$ converge. Then the weak limit is a maximizer by weak lower semicontinuity of the $L^{2}$ norm.

Next, consider any smooth curve $\tau \mapsto y=y(\tau)$ into $H^{2}$ with the property that (3.8) and the no-slip conditions hold for all $\tau$, and $I_{p, k} / I_{u, k}$ achieves its maximum at $\tau=0$. Then at $\tau=0$ we have

$$
0=\dot{I}_{p, k}-\beta_{k} \dot{I}_{u, k}
$$

We now determine $\dot{I}_{p, k}$ and $\dot{I}_{u, k}$ and solve for $\beta_{k}$. Differentiating $I_{p, k}$, we find

$$
\dot{I}_{p, k}=\left(\frac{k^{2}+\alpha^{2}}{k}\right)\left(\overline{\dot{\alpha}}_{+} \alpha_{+}+\bar{\alpha}_{+} \dot{\alpha}_{+}+\overline{\dot{\alpha}}_{-} \alpha_{-}+\bar{\alpha}_{-} \dot{\alpha}_{-}\right)\left(1-|\omega|^{2}\right) .
$$

From (3.8) we infer that

$$
\begin{aligned}
& \alpha_{+}\left(1-\omega^{2}\right)=\left.\partial_{\theta}\left(y_{1}+y_{2}\right) e^{(k+i \alpha)(\theta-\sigma)}\right|_{0} ^{\sigma}, \\
& \alpha_{-}\left(1-\omega^{2}\right)=\left.\partial_{\theta}\left(y_{1}+y_{2}\right) e^{-(k+i \alpha) \theta}\right|_{0} ^{\sigma} .
\end{aligned}
$$

By differentiating in $\tau$, we can solve for $\dot{\alpha}_{+}$and $\dot{\alpha}_{-}$, allowing us to eliminate $\dot{\alpha}_{+}$ and $\dot{\alpha}_{-}$from the formula for $\dot{I}_{p, k}$. Indeed, if we let $\gamma_{i}(\theta)=\partial_{\theta} \dot{y}_{i}$ for $i=1$, 2 , we have

$$
\overline{\dot{\alpha}}_{+}=\left.\frac{\left(1-\omega^{2}\right)}{\left|1-\omega^{2}\right|^{2}}\left(\bar{\gamma}_{1}+\bar{\gamma}_{2}\right) e^{(k-i \alpha)(\theta-\sigma)}\right|_{0} ^{\sigma}, \quad \overline{\dot{\alpha}}_{-}=\left.\frac{\left(1-\omega^{2}\right)}{\left|1-\omega^{2}\right|^{2}}\left(\bar{\gamma}_{1}+\bar{\gamma}_{2}\right) e^{-(k-i \alpha) \theta}\right|_{0} ^{\sigma} .
$$


Similarly, we differentiate $I_{u, k}$. Letting $L_{1}^{*}$ and $L_{2}^{*}$ denote the formal adjoints of $L_{1}$ and $L_{2}$, respectively, and recalling from the no-slip boundary conditions that $\dot{y}=0$ at $\theta=0, \sigma$, we integrate by parts to conclude that

$$
\dot{I}_{u, k}=4\left(\int_{0}^{\sigma} \Re(\rho) d \theta+\Re(\psi)\right),
$$

where

$$
\rho=\overline{\dot{y}}_{1}\left(L_{1}^{*} L_{1}\right) y_{1}+\overline{\dot{y}}_{2}\left(L_{2}^{*} L_{2}\right) y_{2}, \quad \psi=\bar{\partial}_{\theta} \dot{y}_{1}\left(L_{1} y_{1}\right)+\left.\bar{\partial}_{\theta} \dot{y}_{2}\left(L_{2} y_{2}\right)\right|_{0} ^{\sigma} .
$$

We observe that

$$
L_{1} L_{1}^{*} y_{1}=0 \quad \text { and } \quad L_{2} L_{2}^{*} y_{2}=0,
$$

so that $\dot{I}_{u, k}$ reduces to $\dot{I}_{u, k}=4 \Re(\psi)$.

Using this information, we can rewrite (3.12) as

$$
\begin{aligned}
0 & =2 \Re\left\{\left.\bar{\gamma}_{1}\left(2 \beta_{k} L_{1} y_{1}-\left(\frac{k^{2}+\alpha^{2}}{k}\right) \frac{\left(1-|\omega|^{2}\right)}{1-\bar{\omega}^{2}}\left(\alpha_{+} e^{(k-i \alpha)(\theta-\sigma)}+\alpha_{-} e^{-(k-i \alpha) \theta}\right)\right)\right|_{0} ^{\sigma}\right\} \\
& +2 \Re\left\{\left.\bar{\gamma}_{2}\left(2 \beta_{k} L_{2} y_{2}-\left(\frac{k^{2}+\alpha^{2}}{k}\right) \frac{\left(1-|\omega|^{2}\right)}{1-\bar{\omega}^{2}}\left(\alpha_{+} e^{(k-i \alpha)(\theta-\sigma)}+\alpha_{-} e^{-(k-i \alpha) \theta}\right)\right)\right|_{0} ^{\sigma}\right\} .
\end{aligned}
$$

Since $\gamma_{1}(\theta)$ and $\gamma_{2}(\theta)$ are arbitrary at $\theta=0$ and $\theta=\sigma$, (3.14) yields four (natural) boundary conditions:

$$
\begin{aligned}
& 2 \beta_{k} L_{1} y_{1}=\left(\frac{k^{2}+\alpha^{2}}{k}\right) \frac{\left(1-|\omega|^{2}\right)}{1-\bar{\omega}^{2}}\left(\alpha_{+}+\alpha_{-} \bar{\omega}\right) \text { and } \\
& 2 \beta_{k} L_{2} y_{2}=\left(\frac{k^{2}+\alpha^{2}}{k}\right) \frac{\left(1-|\omega|^{2}\right)}{1-\bar{\omega}^{2}}\left(\alpha_{+}+\alpha_{-} \bar{\omega}\right), \text { when } \theta=\sigma, \\
& 2 \beta_{k} L_{1} y_{1}=\left(\frac{k^{2}+\alpha^{2}}{k}\right) \frac{\left(1-|\omega|^{2}\right)}{1-\bar{\omega}^{2}}\left(\alpha_{+} \bar{\omega}+\alpha_{-}\right) \text {and } \\
& 2 \beta_{k} L_{2} y_{2}=\left(\frac{k^{2}+\alpha^{2}}{k}\right) \frac{\left(1-|\omega|^{2}\right)}{1-\bar{\omega}^{2}}\left(\alpha_{+} \bar{\omega}+\alpha_{-}\right), \text {when } \theta=0 .
\end{aligned}
$$

In addition, we have the four no-slip boundary conditions

$$
y_{1}(\sigma)=y_{2}(\sigma)=y_{1}(0)=y_{2}(0)=0 .
$$

3.4. Using (3.16), (3.15), (3.8), and the property $L_{1}^{*} L_{1} y_{1}=L_{2}^{*} L_{2} y_{2}=0$ on $(0, \sigma)$, we can explicitly solve for the maximizer of $\beta_{k}$. To simplify the calculations in what follows, we first use reflection symmetry to show that either

$$
\left(\alpha_{+}, \alpha_{-}\right)=(1,1) \quad \text { or } \quad\left(\alpha_{+}, \alpha_{-}\right)=(1,-1) .
$$

Letting $\hat{\theta}=\sigma-\theta$, we see from our construction of $\hat{q}$ in (3.4) that $\alpha_{+}$and $\alpha_{-}$ exchange roles after reflection; thus, it is natural to set $\hat{\alpha}_{+}=\alpha_{-}$, and $\hat{\alpha}_{-}=\alpha_{+}$. In addition, we let $\hat{y}_{2}(\hat{\theta})=y_{1}(\theta)$ and $\hat{y}_{1}(\hat{\theta})=y_{2}(\theta)$. A straightforward calculation shows that $\left(\hat{y}_{1}, \hat{y}_{2}, \hat{\alpha}_{+}, \hat{\alpha}_{-}\right)$solves the set of linear equations consisting of (3.16), (3.15), (3.8), and $L_{1}^{*} L_{1} y_{1}=L_{2}^{*} L_{2} y_{2}=0$. We deduce that

$\left(y_{1}+\hat{y}_{1}, y_{2}+\hat{y}_{2}, \alpha_{+}+\alpha_{-}, \alpha_{-}+\alpha_{+}\right) \quad$ and $\quad\left(y_{1}-\hat{y}_{1}, y_{2}-\hat{y}_{2}, \alpha_{+}-\alpha_{-}, \alpha_{-}-\alpha_{+}\right)$

also solve these equations. We conclude that every pair $\left(\alpha_{+}, \alpha_{-}\right)$will yield the same value for $\beta_{k}$ as either $\left(\alpha_{+}, \alpha_{-}\right)=(1,1)$ or $\left(\alpha_{+}, \alpha_{-}\right)=(1,-1)$. Therefore it suffices to consider only these cases. 
3.5. We can eliminate $y_{2}$ by observing that if $\left(\alpha_{+}, \alpha_{-}\right)=(1,1)$, then $y_{2}(\theta)=$ $\hat{y}_{2}(\theta)=y_{1}(\sigma-\theta)$, and if $\left(\alpha_{+}, \alpha_{-}\right)=(1,-1)$, then $y_{2}(\theta)=-\hat{y}_{2}(\theta)=-y_{1}(\sigma-\theta)$. Then we infer from boundary conditions in (3.8) that

$$
\begin{aligned}
& 1-\omega=\partial_{\theta} y_{1}(\sigma)-\partial_{\theta} y_{1}(0), \text { when }\left(\alpha_{+}, \alpha_{-}\right)=(1,1), \text { and } \\
& 1+\omega=\partial_{\theta} y_{1}(\sigma)+\partial_{\theta} y_{1}(0), \text { when }\left(\alpha_{+}, \alpha_{-}\right)=(1,-1) .
\end{aligned}
$$

We are now in a position to solve for $y_{1}$ and ultimately for $\beta_{k}$. We first recall that $L_{1}=(i k+1-\alpha)^{2}+\partial_{\theta}^{2}-2 i \partial_{\theta}-1$, while, formally, the adjoint of this operator is given by $L_{1}^{*}=(i k-1+\alpha)^{2}+\partial_{\theta}^{2}-2 i \partial_{\theta}-1$. The characteristic polynomials of these two operators are

$$
\begin{aligned}
& p_{1}(\mu)=(\mu-(2 i-k-i \alpha))(\mu-(k+i \alpha)), \\
& p_{1}^{*}(\mu)=(\mu-(2 i+k-i \alpha))(\mu-(-k+i \alpha)) .
\end{aligned}
$$

Since $L_{1}^{*} L_{1} y_{1}=0$ on $(0, \sigma)$, we can conclude that $y_{1}(\theta)$ takes the form

$$
y_{1}(\theta)=a_{1} e^{(k+i \alpha)(\theta-\sigma)}+a_{2} e^{-(k-2 i+i \alpha) \theta}+a_{3} e^{-(k-i \alpha) \theta}+a_{4} e^{(k+2 i-i \alpha)(\theta-\sigma)}
$$

for some constants $a_{i}, 1 \leq i \leq 4$. The boundary conditions $y_{1}(\sigma)=y_{1}(0)=0$, combined with (3.18), yield the two equalities

$$
\begin{aligned}
& 0=a_{1}+a_{2} \omega e^{2 i \sigma}+a_{3} \bar{\omega}+a_{4}, \\
& 0=a_{1} \omega+a_{2}+a_{3}+a_{4} \bar{\omega} e^{-2 i \sigma} .
\end{aligned}
$$

We will use the boundary conditions for $y_{1}$ in (3.15), combined with the equalities in (3.19), to write the four unknowns $a_{j}, 1 \leq j \leq 4$, in terms of $\alpha_{+}$and $\alpha_{-}$.

Using the equality $L_{j}=L_{j}^{*}+4(1-\alpha) i k$ for $j=1,2$, and (3.18), we conclude that

$$
L_{1} y_{1}=4(1-\alpha) i k\left(a_{3} e^{-(k-i \alpha) \theta}+a_{4} e^{(k+2 i-i \alpha)(\theta-\sigma)}\right) .
$$

Plugging this information into the two boundary conditions in (3.15) yields the two equalities

$$
\begin{aligned}
& 8 \beta_{k}(1-\alpha) i k\left(a_{3} \bar{\omega}+a_{4}\right)=\left(\frac{k^{2}+\alpha^{2}}{k}\right) \frac{\left(1-|\omega|^{2}\right)}{1-\bar{\omega}^{2}}\left(\alpha_{+}+\alpha_{-} \bar{\omega}\right), \\
& 8 \beta_{k}(1-\alpha) i k\left(a_{3}+a_{4} \bar{\omega} e^{-2 i \sigma}\right)=\left(\frac{k^{2}+\alpha^{2}}{k}\right) \frac{\left(1-|\omega|^{2}\right)}{1-\bar{\omega}^{2}}\left(\alpha_{-}+\alpha_{+} \bar{\omega}\right) .
\end{aligned}
$$

3.6. The value of $\beta_{k}$ is determined by the equations in (3.20) and (3.19), together with (3.17). Evidently $\beta_{k}=\max \left\{\beta_{+, k}, \beta_{-, k}\right\}$, where $\beta_{+, k}$ and $\beta_{-, k}$ are the values determined from these equations in each of the two cases $\left(\alpha_{+}, \alpha_{-}\right)=(1,1)$ and $\left(\alpha_{+}, \alpha_{-}\right)=(1,-1)$, respectively.

With $\left(\alpha_{+}, \alpha_{-}\right)=(1,1)$, using the four equations given in (3.20) and (3.19), we solve for the unknowns $a_{j}, 1 \leq j \leq 4$, finding that

$$
\begin{aligned}
& a_{1} \beta_{+, k}\left(1-\omega^{2} e^{2 i \sigma}\right)=-\phi_{1}\left(1-\omega e^{2 i \sigma}\right), \\
& a_{2} \beta_{+, k}\left(1-\omega^{2} e^{2 i \sigma}\right)=-\phi_{1}(1-\omega), \\
& a_{3} \beta_{+, k}\left(1-\bar{\omega}^{2} e^{-2 i \sigma}\right)=\phi_{1}\left(1-\bar{\omega} e^{-2 i \sigma}\right), \\
& a_{4} \beta_{+, k}\left(1-\bar{\omega}^{2} e^{-2 i \sigma}\right)=\phi_{1}(1-\bar{\omega}),
\end{aligned}
$$

where

$$
\phi_{1}=\frac{\left(k^{2}+\alpha^{2}\right)\left(1-|\omega|^{2}\right)(1+\bar{\omega})}{8 i k^{2}(1-\alpha)\left(1-\bar{\omega}^{2}\right)}
$$


Using (3.17) with (3.18), we see that

$$
\begin{aligned}
1-\omega= & a_{1} \hat{k}(1-\omega)+a_{2}(2 i-\hat{k})\left(\omega e^{2 i \sigma}-1\right) \\
& +a_{3}(\overline{\hat{k}})(1-\bar{\omega})+a_{4}(2 i+\overline{\hat{k}})\left(1-\bar{\omega} e^{-2 i \sigma}\right),
\end{aligned}
$$

where $\hat{k}=k+i \alpha$. Plugging the formulas for $a_{j}$ into (3.22) and solving for $\beta_{+, k}$ yields

$$
\beta_{+, k}=\frac{\phi_{1}}{(1-\omega)}\left\{\frac{(1-\omega)\left(1-\omega e^{2 i \sigma}\right)}{\left(1-\omega^{2} e^{2 i \sigma}\right)}(2 i-2 \hat{k})+\frac{(1-\bar{\omega})\left(1-\bar{\omega} e^{-2 i \sigma}\right)}{\left(1-\bar{\omega}^{2} e^{-2 i \sigma}\right)}(2 i+2 \overline{\hat{k}})\right\} .
$$

To find $\beta_{-, k}$, we let $\left(\alpha_{+}, \alpha_{-}\right)=(1,-1)$, and we again use (3.20) and (3.19) to solve for $a_{j}, 1 \leq j \leq 4$. To simplify notation, we define

$$
\phi_{2}=\frac{\left(k^{2}+\alpha^{2}\right)\left(1-|\omega|^{2}\right)(1-\bar{\omega})}{8 i k^{2}(1-\alpha)\left(1-\bar{\omega}^{2}\right)} .
$$

We compute the $a_{j}$ and conclude that

$$
\begin{aligned}
& a_{1} \beta_{-, k}\left(1-\omega^{2} e^{2 i \sigma}\right)=-\phi_{2}\left(1+\omega e^{2 i \sigma}\right), \\
& a_{2} \beta_{-, k}\left(1-\omega^{2} e^{2 i \sigma}\right)=\phi_{2}(1+\omega), \\
& a_{3} \beta_{-, k}\left(1-\bar{\omega}^{2} e^{-2 i \sigma}\right)=-\phi_{2}\left(1+\bar{\omega} e^{-2 i \sigma}\right), \\
& a_{4} \beta_{-, k}\left(1-\bar{\omega}^{2} e^{-2 i \sigma}\right)=\phi_{2}(1+\bar{\omega}) .
\end{aligned}
$$

We solve for $\beta_{-, k}$ using (3.17) with (3.18) as before, and we find that

$$
\beta_{-, k}=\frac{\phi_{2}}{(1+\omega)}\left\{\frac{(1+\omega)\left(1+\omega e^{2 i \sigma}\right)}{\left(1-\omega^{2} e^{2 i \sigma}\right)}(2 i-2 \hat{k})+\frac{(1+\bar{\omega})\left(1+\bar{\omega} e^{-2 i \sigma}\right)}{\left(1-\bar{\omega}^{2} e^{-2 i \sigma}\right)}(2 i+2 \overline{\hat{k}})\right\} .
$$

At this point, one can check that $\beta_{ \pm, k}=\hat{\beta}_{ \pm, k}$, as given in (1.9).

3.7. To complete the proof of Theorem [1.4 as indicated at the beginning of this section, we must show that $\beta_{\sigma, \alpha} \geq \hat{\beta}_{\sigma, \alpha}$. To prove this, suppose $\hat{\beta}<\hat{\beta}_{\sigma, \alpha}$. Then there exists $k_{0} \neq 0$ such that $\beta_{k_{0}}>\hat{\beta}$. We choose $y$ to be a maximizer of the ratio $I_{p, k_{0}} / I_{u, k_{0}}$. In a change of notation, we let $I_{p, k}$ and $I_{u, k}$ denote the integrals corresponding to this fixed $y$, with $\hat{q}$ determined by (2.9) for $k$ varying. Since $y$ may not be a maximizer for $k \neq k_{0}$, we only have $\beta_{k} \geq I_{p, k} / I_{u, k}$ in general. However, by continuity it is evident that there exists $\delta>0$ such that whenever $\left|k-k_{0}\right|<\delta$ we have $I_{p, k} / I_{u, k}>\hat{\beta}$.

Next, we define $\chi_{\delta}(k)$ to be a smooth bump function independent of $\theta$ and supported in a $\delta$-neighborhood of $k_{0}$. Recalling that $-i \hat{w}=V y$, we set $\hat{w}_{\delta}=\chi_{\delta} \hat{w}$ and $\hat{q}_{\delta}=\chi_{\delta} \hat{q}$, and we observe that $\left(\hat{w}_{\delta}, \hat{q}_{\delta}\right)$ solves (2.9) and $\hat{w}_{\delta}=0$ for $\theta=0, \sigma$. Moreover, if $I_{p_{\delta}, k}$ and $I_{u_{\delta}, k}$ are the integrals corresponding to $\hat{w}_{\delta}$ and $\hat{q}_{\delta}$, then one sees that $I_{p_{\delta}, k}=\chi_{\delta}^{2} I_{p, k}$ and $I_{u_{\delta}, k}=\chi_{\delta}^{2} I_{u, k}$. We can then write

$$
\frac{I_{p_{\delta}}}{I_{u_{\delta}}}=\frac{\int_{k_{0}-\delta}^{k_{0}+\delta} \chi_{\delta}^{2} I_{p, k} d k}{\int_{k_{0}-\delta}^{k_{0}+\delta} \chi_{\delta}^{2} I_{u, k} d k}>\frac{\int_{k_{0}-\delta}^{k_{0}+\delta} \hat{\beta} \chi_{\delta}^{2} I_{u, k} d k}{\int_{k_{0}-\delta}^{k_{0}+\delta} \chi_{\delta}^{2} I_{u, k} d k}=\hat{\beta} .
$$

We conclude that $\beta_{\sigma, \alpha} \geq \hat{\beta}$; hence $\beta_{\sigma, \alpha} \geq \hat{\beta}_{\sigma, \alpha}$. 


\section{Causes for Blowup of the optimal CONSTANT}

One can rewrite the formulas for $\hat{\beta}_{+, k}$ and $\hat{\beta}_{-, k}$ from Theorem 1.4 in the following way:

$$
\hat{\beta}_{ \pm, k}=\frac{\psi_{1}+\psi_{2}}{2 k^{2}(\cosh (k \sigma) \mp \cos (\alpha \sigma))(\cosh (2 k \sigma)-\cos (2(1-\alpha) \sigma))},
$$

where

$$
\psi_{1}=\left(k^{2}+\alpha^{2}\right) \sinh (k \sigma)[\sinh (2 k \sigma) \mp 2 \sinh (k \sigma) \cos (\sigma) \cos ((1-\alpha) \sigma)]
$$

and

$$
\psi_{2}=\frac{k\left(k^{2}+\alpha^{2}\right) \sinh (k \sigma)}{1-\alpha}[\sin (2(1-\alpha) \sigma) \mp 2 \cosh (k \sigma) \sin ((1-\alpha) \sigma) \cos (\sigma)] .
$$

From (4.1) it is clear that for fixed $\alpha \neq 1$ and fixed $\sigma \in(0,2 \pi), \hat{\beta}_{+, k}$ and $\hat{\beta}_{-, k}$ as functions of $k$ are continuous everywhere except $k=0$. If we take the limit of (4.1) as $k$ approaches 0 , we find that

$$
\lim _{k \rightarrow 0} \hat{\beta}_{ \pm, k}=\hat{\beta}_{ \pm, 0}=\frac{\alpha^{2} \psi_{3}}{2(1 \mp \cos (\alpha \sigma))(1-\cos 2(1-\alpha) \sigma)},
$$

where

$$
\begin{aligned}
\psi_{3}= & 2 \sigma^{2} \mp \sigma^{2}(\cos (\alpha \sigma)+\cos (2-\alpha) \sigma) \\
& -\frac{\sigma}{1-\alpha}(\sin (2-2 \alpha) \sigma \mp(\sin (2-\alpha) \sigma-\sin (\alpha \sigma))) .
\end{aligned}
$$

From (4.2) we see that $\beta_{\sigma, \alpha}$ typically blows up when either $\alpha \sigma=n \pi$ or $(1-\alpha) \sigma=n \pi$ for some $n \in \mathbb{Z}$.

The first set of singularities above is a result of the unboundedness of the Neumann problem for the Laplace operator in weighted spaces on a cone. To see this, we observe that in (3.13) $\alpha_{+}$and $\alpha_{-}$become undefined as $k \rightarrow 0$ when $\omega^{2}=e^{-2(k+i \alpha) \sigma} \rightarrow 1$, which occurs precisely when $\alpha \sigma=n \pi$ for $n \in \mathbb{Z}$.

The second set of singularities above, which occur when $(1-\alpha) \sigma=n \pi$, result from failure to bound the boundary data $n \cdot(\Delta-\nabla \nabla \cdot) u$ in terms of $\Delta u$. For these combinations of $\alpha$ and $\sigma$, the $L^{2}$ norm of $r^{\alpha} \Delta u$ in $\mathcal{K}_{\sigma}$ is not sufficient to control $n \cdot(\Delta-\nabla \nabla \cdot) u$ appropriately. This is fundamentally due to the existence of harmonic fields $u=c r^{1-\alpha} \sin ((1-\alpha) \theta)$ where $c$ is a constant vector. Corresponding to these fields, there are nontrivial modes $\left(y_{1}, y_{2}\right)$ for $k=0$ satisfying $L_{1} y_{1}=0=L_{2} y_{2}$ and the no-slip boundary conditions (3.16), while $\left(\alpha_{+}, \alpha_{-}\right)$is nonzero. One then finds that the maximum of $I_{p, k} / I_{u, k} \rightarrow \infty$ as $k \rightarrow 0$.

To see just how this can occur in terms of the computations of Section 3 for certain combinations of $\sigma$ and $\alpha(\neq 1$ or 0$)$, we observe that in Section $3.5 L_{1} y_{1}=0$ iff $y_{1}(\theta)$ takes the form given in (3.18) with $a_{3}=a_{4}=0$. One can then satisfy the no-slip boundary conditions through (3.19) for some nonzero $a_{1}, a_{2}$ if and only if $\omega^{2} e^{2 i \sigma}=1$, meaning $k=0$ and $(1-\alpha) \sigma=n \pi$ for some $n \in \mathbb{Z}$. We may simply take $y_{2}=0$, and it follows by (3.3) that $I_{u, 0}=0$.

But then, $a_{1}=-a_{2} \bar{\omega} \neq 0$, and we compute that $\partial_{\theta} y_{1}=2 i(\alpha-1) a_{1} \neq 0$ at $\theta=\sigma$, yielding nonzero boundary values for $\partial_{\theta} \hat{q}$ in (3.7) and causing $I_{p, 0}$ to be positive in (2.3). If we vary $k$ while holding $\left(y_{1}, y_{2}\right)$ fixed and use (3.8) to determine $\left(\alpha_{+}, \alpha_{-}\right)$ and thence $\hat{q}$, we see that $I_{u, k} \rightarrow 0$ as $k \rightarrow 0$, while $I_{p, k} \rightarrow I_{p, 0}>0$. This results in $\beta_{k} \rightarrow \infty$ as $k \rightarrow 0$; hence $\beta_{\sigma, \alpha}=\infty$ when $(1-\alpha) \sigma=n \pi$. 


\section{Proof of Theorem 1.2}

We now use Theorem 1.4 to prove Theorem 1.2 through a localization argument. Let $\Omega$ denote a bounded domain with a straight corner. Replacing $\Omega$ by a suitable rotated translate if necessary, we may assume there is a neighborhood $U$ of 0 such that $U \cap \Omega=U \cap \mathcal{K}_{\sigma}$, where $\sigma \neq \pi$.

Fix any $\beta<1$ and $C \in \mathbb{R}$. We observe from the formula for $\hat{\beta}_{ \pm, k}$ with $\alpha=0$ given in (1.10) that $\beta_{\sigma, 0} \geq 1$ when $\sigma \neq \pi$. Therefore, there exists a solution $(u, p)$ to (2.8) with $u$ in $C_{c}^{\infty}\left(\overline{\mathcal{K}}_{\sigma} \backslash\{0\}, \mathbb{R}^{2}\right)$ which satisfies $\int_{\mathcal{K}_{\sigma}}|\nabla p|^{2}>\beta \int_{\mathcal{K}_{\sigma}}|\Delta u|^{2}$. Replacing $(u, p)$ by suitable dilates if necessary, we may assume that the support of $u$ is contained in $U$.

We construct a sequence of solutions $\left(u_{j}, p_{j}\right)$ to (2.8) on $\Omega$ by setting

$$
u_{j}(x)=\left.j^{-1} u(j x)\right|_{\Omega} \text { and } \nabla p_{j}=(I-P)(\Delta-\nabla \nabla \cdot) u_{j} \text { in } \Omega .
$$

We see that $\Delta p_{j}=0$ in $\Omega$ and $n \cdot \nabla p_{j}=n \cdot(\Delta-\nabla \nabla \cdot) u_{j}$ on $\partial \Omega$. Moreover, since $u_{j}$ is supported in $\Omega \cap \mathcal{K}_{\sigma}$ for all $j$, we have $\left\|\Delta u_{j}\right\|_{L^{2}(\Omega)}=\|\Delta u\|_{L^{2}(j \Omega)} \leq\|\Delta u\|_{L^{2}\left(\mathcal{K}_{\sigma}\right)}$ and $\left\|\nabla u_{j}\right\|_{L^{2}(\Omega)}=j^{-1}\|\nabla u\|_{L^{2}(j \Omega)} \leq j^{-1}\|\nabla u\|_{L^{2}\left(\mathcal{K}_{\sigma}\right)}$ for every $j$. This construction allows us to write the following series of inequalities for sufficiently small $\epsilon>0$ and for sufficiently large $j$ :

$$
\begin{gathered}
\int_{\Omega}\left(\beta\left|\Delta u_{j}\right|^{2}+C\left|\nabla u_{j}\right|^{2}\right) \leq \int_{\mathcal{K}_{\sigma}}\left(\beta|\Delta u|^{2}+C j^{-2}|\nabla u|^{2}\right) \\
\leq \int_{\mathcal{K}_{\sigma}}\left(\beta|\Delta u|^{2}\right)+\epsilon<\int_{\mathcal{K}_{\sigma}}|\nabla p|^{2} .
\end{gathered}
$$

We claim that

$$
\int_{\mathcal{K}_{\sigma}}|\nabla p|^{2} \leq \liminf _{j \rightarrow \infty} \int_{\Omega}\left|\nabla p_{j}\right|^{2}
$$

To see that (5.1) holds, we first use the definition of $u_{j}$, the equality

$$
\nabla p_{j}=(I-P)(\Delta-\nabla \nabla \cdot) u_{j},
$$

and orthogonality of the Leray projection to observe that

$$
\int_{\Omega}\left|\nabla p_{j}\right|^{2} \leq \int_{j \Omega}|(\Delta-\nabla \nabla \cdot) u|^{2} \leq \int_{\mathcal{K}_{\sigma}}|(\Delta-\nabla \nabla \cdot) u|^{2}
$$

for all $j$. If we define

$$
p_{j}^{*}(x)=p_{j}\left(\frac{x}{j}\right)-\frac{1}{m(B)} \int_{B} p_{j}\left(\frac{x}{j}\right)
$$

for $x \in j \Omega$, where $B$ corresponds to the domain $B$ given in (1.7), then we can apply a generalized Poincaré Inequality (see [5, Ch. 2]) to conclude that for each $n \in \mathbb{N}$,

$$
\int_{n \Omega}\left|p_{j}^{*}\right|^{2} \leq C_{n} \int_{n \Omega}\left|\nabla p_{j}^{*}\right|^{2} \leq C_{n} \int_{\Omega}\left|\nabla p_{j}\right|^{2}
$$

for sufficiently large $j$. By a standard diagonalization argument, we can construct a subsequence of $\left\{\nabla p_{j}^{*}\right\}$, which we henceforth denote as $\left\{\nabla p_{j}^{*}\right\}$, converging weakly in $L^{2}(n \Omega)$ for every $n \in \mathbb{N}$. This implies by (5.4) and by another diagonalization 
argument that, up to subsequences, $\left\{p_{j}^{*}\right\}$ converges weakly to some $p^{*}$ in $L^{2}(n \Omega)$ for all $n \in \mathbb{N}$. By uniqueness of weak limits, we can conclude that $\left\{\nabla p_{j}^{*}\right\}$ converges weakly to $\nabla p^{*}$ in $L^{2}(n \Omega)$ for every $n$. Moreover, by properties of weakly convergent sequences we can write $\int_{n \Omega}\left|\nabla p^{*}\right|^{2} \leq \liminf _{j \rightarrow \infty} \int_{n \Omega}\left|\nabla p_{j}^{*}\right|^{2}$ for each $n$. We can then conclude that for sufficiently large $j$,

$$
\int_{\mathcal{K}_{\sigma}}\left|\nabla p^{*}\right|^{2} \leq \lim _{n \rightarrow \infty} \int_{n \Omega}\left|\nabla p^{*}\right|^{2} \leq \liminf _{j \rightarrow \infty} \int_{j \Omega}\left|\nabla p_{j}^{*}\right|^{2}=\liminf _{j \rightarrow \infty} \int_{\Omega}\left|\nabla p_{j}\right|^{2} .
$$

It remains to show that $\int_{\mathcal{K}_{\sigma}}\left|\nabla p^{*}\right|^{2}=\int_{\mathcal{K}_{\sigma}}|\nabla p|^{2}$. This will imply (5.1).

To show that $\int_{\mathcal{K}_{\sigma}}\left|\nabla p^{*}\right|^{2}=\int_{\mathcal{K}_{\sigma}}|\nabla p|^{2}$, we first show $\Delta p^{*}=0$ in $\mathcal{K}_{\sigma}$. We fix a compact subset $K$ of $\mathcal{K}_{\sigma}$, and we apply the mean value property and weak convergence of $\left\{p_{j}^{*}\right\}$ to conclude that for any $y \in K,\left|p_{j}^{*}(y)\right| \leq C\left\|p_{j}^{*}\right\|_{L^{2}(n \Omega)} \leq C$, giving equiboundedness of $\left\{p_{j}^{*}\right\}$ on $K$. Moreover, by the mean value property and weak convergence of $\left\{\nabla p_{j}^{*}\right\},\left\{\nabla p_{j}^{*}\right\}$ is equibounded on $K$, implying that $\left\{p_{j}^{*}\right\}$ is also equicontinuous. Therefore, up to subsequences, $\left\{p_{j}^{*}\right\}$ converges uniformly on $K$ to $p^{*}$. We again apply the mean value property and uniform convergence of $\left\{p_{j}^{*}\right\}$ on $K$ to conclude that $p^{*}$ is harmonic.

Since $\Delta p^{*}=0$ on $n \Omega$, we infer that the sequence $\left\{\nabla p_{j}^{*}\right\}$ converges weakly to $\nabla p^{*}$ in $H(\operatorname{div}, n \Omega)$, the space of vector fields in $L^{2}(n \Omega)$ with divergence in $L^{2}(n \Omega)$. By the boundedness of the trace operator mapping $H(\operatorname{div}, n \Omega)$ into $H^{-\frac{1}{2}}(\partial(n \Omega))$ (see, for example, 1, Theorem 2.5), we can conclude that $n \cdot \nabla p_{j}^{*}$ converges weakly to $n \cdot \nabla p^{*}$ in $H^{-\frac{1}{2}}(\partial(n \Omega))$. As $n \cdot \nabla p_{j}^{*}=n \cdot \nabla p$ on $\partial \mathcal{K}_{\sigma} \cap \partial(n \Omega)$ for every $n$, it follows that $n \cdot \nabla p^{*}=n \cdot \nabla p$ on $\partial \mathcal{K}_{\sigma}$.

Using the equalities $\Delta p=\Delta p^{*}=0$ in $\mathcal{K}_{\sigma}$ and $n \cdot \nabla\left(p^{*}-p\right)=0$ on $\partial \mathcal{K}_{\sigma}$, we can now integrate by parts to conclude that $\int_{\mathcal{K}_{\sigma}}\left|\nabla\left(p^{*}-p\right)\right|^{2}=0$. For $\phi \in C_{c}^{\infty}\left(\overline{\mathcal{K}}_{\sigma}\right)$, we have that

$$
\int_{\mathcal{K}_{\sigma}} \nabla \phi \cdot \nabla\left(p^{*}-p\right)=\int_{\partial \mathcal{K}_{\sigma}} \phi n \cdot \nabla\left(p^{*}-p\right)-\int_{\mathcal{K}_{\sigma}} \phi \Delta\left(p^{*}-p\right)=0 .
$$

Since $p^{*}-p$ belongs to $Y$ and $C_{c}^{\infty}\left(\overline{\mathcal{K}}_{\sigma}\right)$ is dense in $Y$, it follows that

$$
\int_{\mathcal{K}_{\sigma}}\left|\nabla\left(p^{*}-p\right)\right|^{2}=0
$$

and (5.1) holds. This completes the proof of Theorem 1.2 .

\section{REFERENCES}

1. V. Girault and P.-A. Raviart. Finite Element Methods for Navier-Stokes Equations. Theory and Algorithms. Springer Series in Computational Mathematics, 5. Springer, Berlin, 1986. MR.851383 (88b:65129)

2. V. A. Kozlov, V. G. Maz'ya, and J. Rossmann. Elliptic Boundary Value Problems in Domains with Point Singularities. Surveys and Monographs, 52, Amer. Math. Soc., Providence, RI, 1997. MR.1469972 (98f:35038)

3. J.-G. Liu, J. Liu, and R. Pego. Stability and Convergence of Efficient Navier-Stokes Solvers via a Commutator Estimate. Comm. Pure Appl. Math., 60:1443-1487, 2007. MR2342954 (2008k:76039) 
4. R. Rostamian and A. M. Soane. Variational Problems in Weighted Sobolev Spaces on Nonsmooth Domains, Quart. Appl. Math., 68:439-458, 2010.

5. H. Sohr. The Navier-Stokes Equations. An Elementary Functional Analytic Approach. Birkhäuser Advanced Texts: Basler Lehrbücher, Birkhäuser, Basel, 2001. MR.1928881 (2004b:35265)

Department of Mathematical Sciences, Carnegie Mellon University, Pittsburgh, PenNSYlVania 15213-3890

E-mail address: ecozzi@andrew.cmu.edu

Current address: Department of Mathematics, Drexel University, Philadelphia, Pennsylvania 19104

E-mail address: ecozzi@drexel.edu

Department of Mathematical Sciences, Carnegie Mellon University, Pittsburgh, PEnNSYlVANia 15213-3890

E-mail address: rpego@andrew.cmu.edu 